\title{
ANWENDUNG EINES GENETISCHEN ALGORITHMUS AUF DAS SWAPPING-PROBLEM
}

\section{Carl-Heinz Meyer, FernUniversität Hagen}

Bei der Steuerung von Roboter-Greifarmen tritt das folgende Problem auf:

Auf einer Ebene seien $n$ Gegenstände an bestimmten Punkten aufgestellt. $\mathrm{Zu}$ jedem Gegenstand gibt es eine gewünschte Endposition, in die dieser mit Hilfe eines Roboter-Greifarmes bewegt werden soll. Leider kann der Greifarm lediglich einen Gegenstand aufnehmen und zu einem neuen Punkt transportieren. Das Problem besteht nun darin, einen kürzesten Weg für den Greifarm zu ermitteln, sodaß alle Gegenstände ohne zwischenzeitliches Absetzen an ihre gewünschte Positionen überführt werden. (Man stelle sich ein gerade benutztes Schachbrett vor, dessen Figuren wieder in ihre Ausgangsposition gebracht werden sollen) Genetische Algorithmen gehören zur Klasse der stochastischen parallelen Verfahren, mit denen sich auch für große NP-harte Probleme oftmals gute suboptimale Lösungen auffinden lassen. Die Problemstellung wird hierbei durch Mengen (Generationen) von binären Strings mit einer festen Länge repräsentiert. Aus diesen Generationen werden einige Strings für die Auswahl von Nachfolgegenerationen ausgewählt, wobei die ausgewählten Strings nach bestimmmten Regeln verändert werden. Sowohl die Auswahl als auch die Veränderungen orientieren sich nach Prinzipien, die auch in der Natur Verwendung finden, wie z.B. "Darvinian Survival of the Fittest" bzw. "Mutationen" unc "Crossover"

Dieser Artikel beschreibt die Anwendung sowie einige Simulationsergebnisse von Genetischen Algorithmen bei der Lösung des Swapping-Problrms. 